\title{
Using Raster Based Solutions to Identify Spatial Economic Agglomerations
}

\author{
Marian DÂRDALĂ, Adriana REVEIU \\ Bucharest Academy of Economic Studies, Romania \\ dardala@ase.ro,reveiua@ase.ro
}

The efficient economic activities incline to be concentrated in space, and therefore there is an increased attention over the forces of spatial economic agglomerations and the role of location in economic development. This paper proposes some solutions to automate the identification of spatial agglomerations and their intensities, function on the spatial distribution of items in the geographical areas. The software components developed to accomplish this task are Geographic Information Systems specific tools.

Keywords: GIS, Spatial Agglomerations, Raster, Framework, Raster Symbology

1 Introduction

There are a lot of scientific papers supporting the idea that in the presence of agglomeration economies, the potential for growth is increasing in the level of economic activity [1].

The huge amount of spatial data generated by GIS (Geographical Information Systems) expansion, the increasing number of geographic informatics applications available, the computerization of a large amount of information sources, and the availability of digital map has increased the opportunity and need for the usage of methods and techniques for spatial data analysis and integration with economic data, for both research and applied purposes. [3]

This paper proposes a GIS based solution to automate the identification of agglomerations in space, by determining the density of spatial elements, function on their physical locations.

The proposed solutions suppose to use software components developed to automate and to reuse the same behavior, for a large set of applications, developed for many domains and economic applications. The software components could be used in scenarios based manner.

Scenarios developed in a certain field, can be reused in the same domain, extending or improving them, or in other scientific fields, by adapting them to new requirements. The process of reuse is dependent on the complexity of the information presented, describing the use of functions implemented readiness of users in areas that have been developed for these scenarios. [5]

The proposed solutions use raster concept, as support to generate the level of density of spatial distributed economic items, i.e. the density of companies performing in one economic field, as they are distributed in space and the distribution of communication means like roads, in space, based on their densities.

A raster consists of a matrix of cells, each containing a value representing information, such as: temperature or the presence of one economic phenomenon in a specific place.

Rasters are digital aerial photographs, imagery from satellites, digital pictures, or even scanned maps.

Data stored in a raster format represents realworld phenomena, like: thematic or discrete data, representing features such as land-use or soils data and continuous data, representing phenomena such as temperature, elevation or spectral data such as satellite images and aerial photographs.

Within a GIS, the uses of raster data fall under the following main categories:

- raster as a basemap by using orthophotographs - it is a background display for other feature layers. This kind of raster is used to display underneath other layers, and provide the map users with the confidence that map layers are spatially aligned and represent real objects, together with representing of additional information. There are three main sources of raster basemaps: from aerial photography, satellite imagery, and scanned maps; 
- raster as a surface map -are well suited rasters for representing data that changes continuously across a surface. They provide an effective method of storing the continuity as a surface. They also provide a regularly spaced representation of surfaces. Elevation values measured from the earth's surface are the most common application of surface maps, but other values, such as rainfall, temperature, concentration, and population density, can also define surfaces that can be spatially analyzed;

- raster as a thematic map - are raster representing thematic data, which can be derived from analyzing other data. Thematic maps can result from geoprocessing operations, which combine data from various sources, such as vector, raster, and terrain data. For example, you can process data through a geoprocessing model to create a raster dataset that maps suitability for a specific activity;

- raster as attributes of a feature - rasters used as attributes of a feature may be digital photographs, scanned documents, or scanned drawings related to a geographic object or location[6].

The main advantages of storing the spatial data as a raster are: the simple data structure because a matrix of cells with values representing a coordinate and sometimes linked to an attribute table; a powerful format for ad- vanced spatial and statistical analysis; the ability to represent continuous surfaces and to perform surface analysis; the ability to uniformly store points, lines, polygons, and surfaces; and the ability to perform fast overlays with complex datasets. [6]

\section{Programmatic Solutions}

To accomplish the tasks of this paper, solutions based on the raster processing are used. To develop the software components the ArcObjects framework is used. ArcObjects is a framework developed on COM (component) type technology, used to build ArcGIS software, developed by ESRI (Environmental Systems Research Institute) [4].

By using a framework to develop software applications, the advantage that allows developers to reuse code and also to facilitate the building of new extensions is supported. In order to use the raster based symbology, that allow us to reflect the spatial data density in the territorial area, a hierarchy of classes has been developed. The hierarchy of classes enables the use of specialized coclasses for generating the symbology, based on spatial data types like point and polyline. The coclasses are used to generate new objects [3].

Figure 1 presents the developed of classes hierarchy.

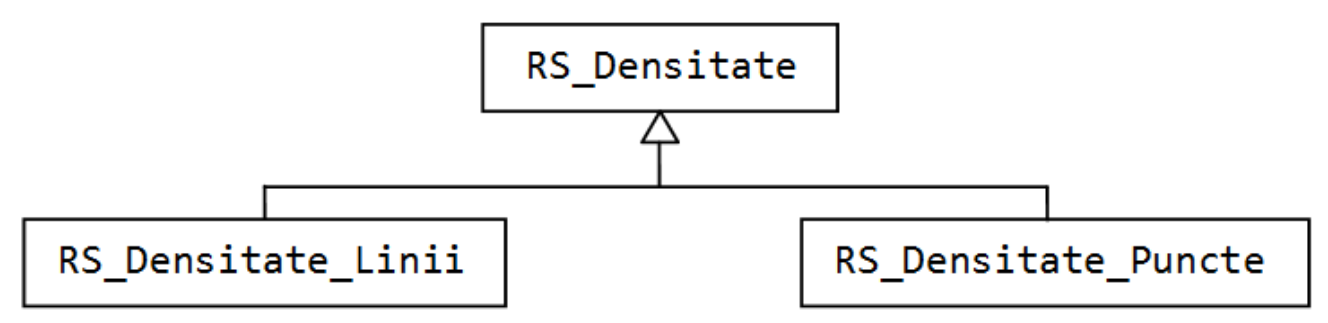

Fig. 1. The hierarchy of classes used to display spatial data density in space

In ArcObjects, the raster is organized as a matrix of values, having different types: byte, int, double, and so on, and it is organized into levels. A level contains a matrix which, semantically, expresses something. For example, if we include in ArcMap a bitmap image, built based on RGB model, as a thematic layer, it will be held as having three layers, with three arrays of values: one matrix of values represents red color, one with values for green color, and the third with values for blue color. A raster point is displayed, from chro- 
matic point of view, like a combination of the three values: the values of red, green and blue layers. Figure 2 illustrates this structure for an image having $m \times n$ resolution.

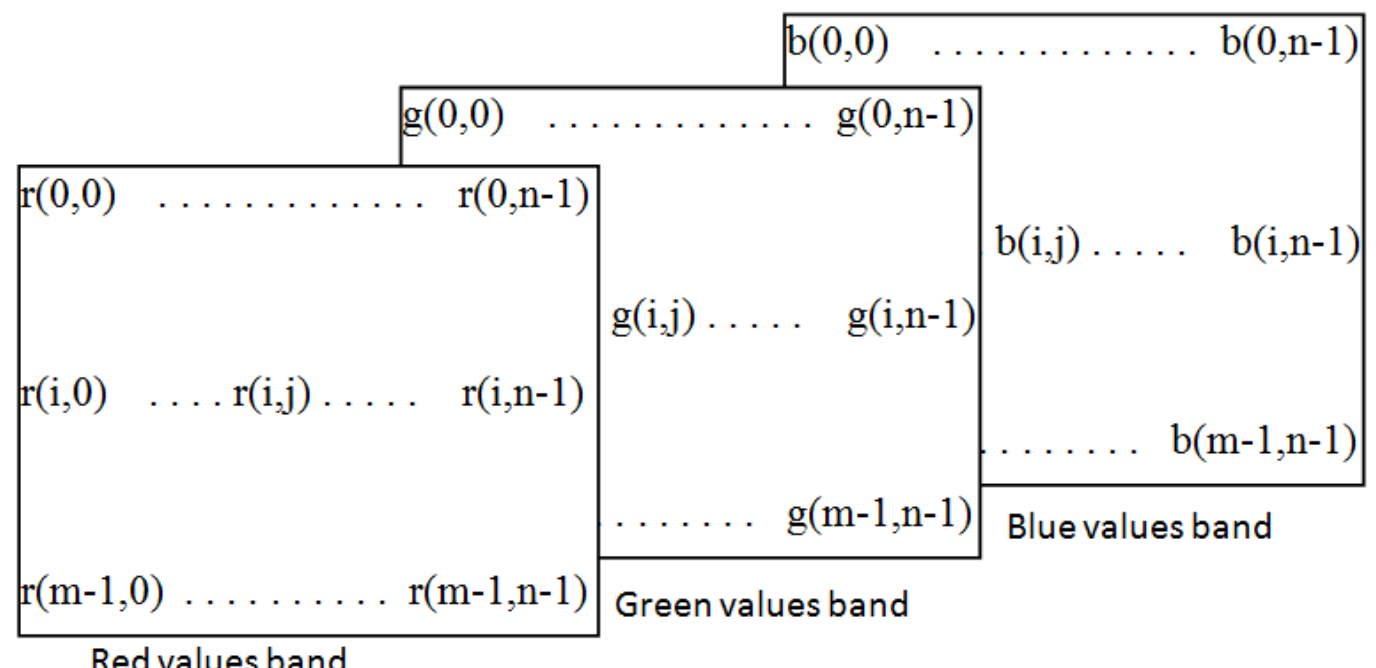

$$
\operatorname{Pixel}(i, j)=R G B(r(i, j), g(i, j), b(i, j))
$$

Fig. 2. The decomposition of one image into three bands, represented in RGB

Considering the terminology used by the ArcObjects framework, two coclasses has been defined (RS_Densitate_Linii and $R S \_$Densitate_Puncte), and an abstract class (RS_Densitate) having the goal to close the hierarchy. $R S \_$Densitate class contains working elements used by the two coclasses. It is an inheritance relationship between classes, in the sense that coclasses inherit attributes and methods of the base abstract class. Raster type symbology can be generated, from spatial data having specific types like: line or point. $R S \_$Densitate class has been defined as an abstract class, because it cannot be directly instantiate. The Generare_Raster method used to generate the raster has been described as a pure virtual method because it cannot be concretized inside the abstract class, but it makes sense to be defined the concrete classes derived from it. Generare_Raster method aims to generate raster, by using the input parameters, some are common for both types of spatial data, and others are specific for each type of data. Once obtained, the raster can be appropriate symbolized, so that the areas from geographic studied are able to be displayed as suggestive as possible, in terms of density of spatial data generated. Because the symbology applies to the resulting raster, the specific methods used to generate the symbology were grouped into parent class (the abstract one). For this purpose three methods have been defined:

- Afisare_Implicita used to display the raster by default, i.e. by using colors from black to white; the black color is associated with the lowest density areas, and white color is associated with the highest density areas, intermediate density areas are coloured in shades of gray;

- Afisare_Continua used to display the raster by using continuous tone of colors, the user provides, for this purpose, the start color that will match the lower density areas and the last color has to be associated with the highest density areas; intermediate areas will be colored with transitional tones, that make the transition from initial to final colors;

- Afisare_Discreta used discrete color tones to display the raster. The user can define the number of groups used to divide the area, according to the calculated densities. Thus, a number of intervals of values have 
to be set out, for the density values, and a color will be assigned for each group (range) of values to view these areas. In this case, the extreme colors will be provided, and the intermediate groups will be then filled with transition colors.

To have a high degree of generality, for the hierarchy of classes, a constructor has been defined. The constructor has the goal to create the connection with the document map, which is generated based on raster symbology. The input parameters are provided in the form of properties, which are of read-write type.

The abstract class RS_Densitate has the following content:

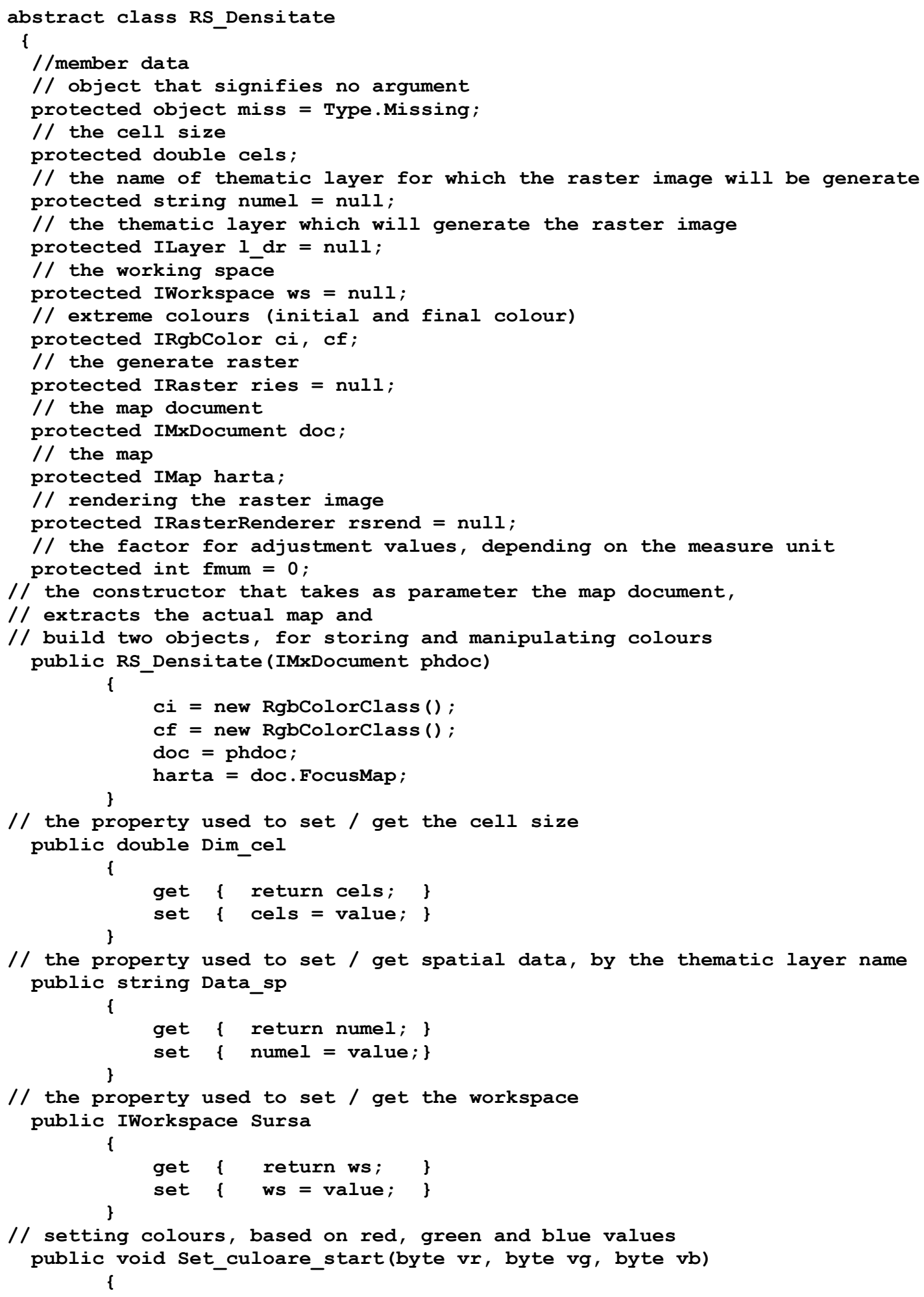




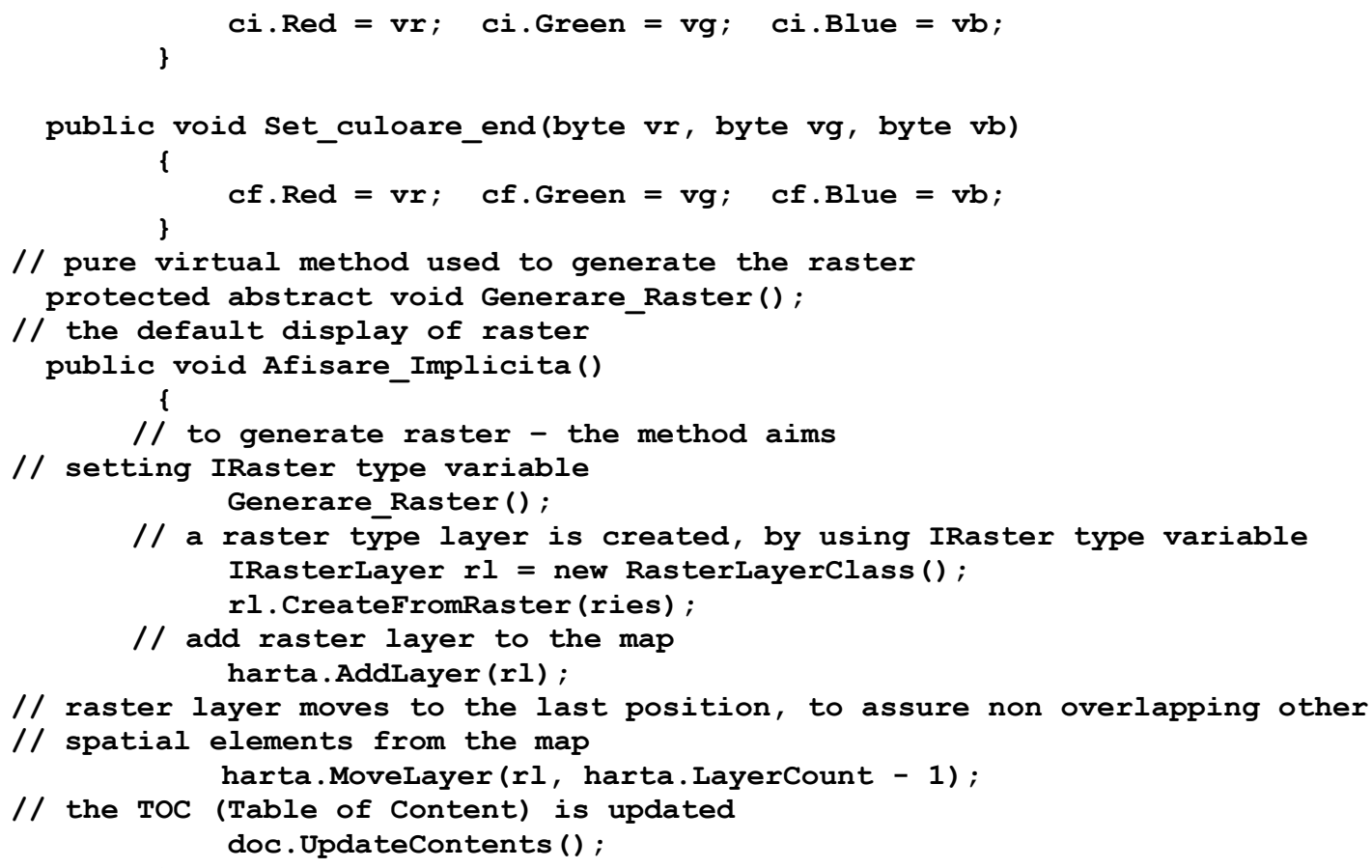

To display the density of spatial data like a raster, a raster with only one level has to be developed. The matrix stores the density values, in the required points, so that on displaying, the numeric values will be scaled on a color ramp. Thus, the points turn differently accuracy in representing the densities, the raster has to contain an array of real values (double).

Get_minmax method aims to calculate the minimum and maximum values of the raster matrix. depending on their values. To have a better

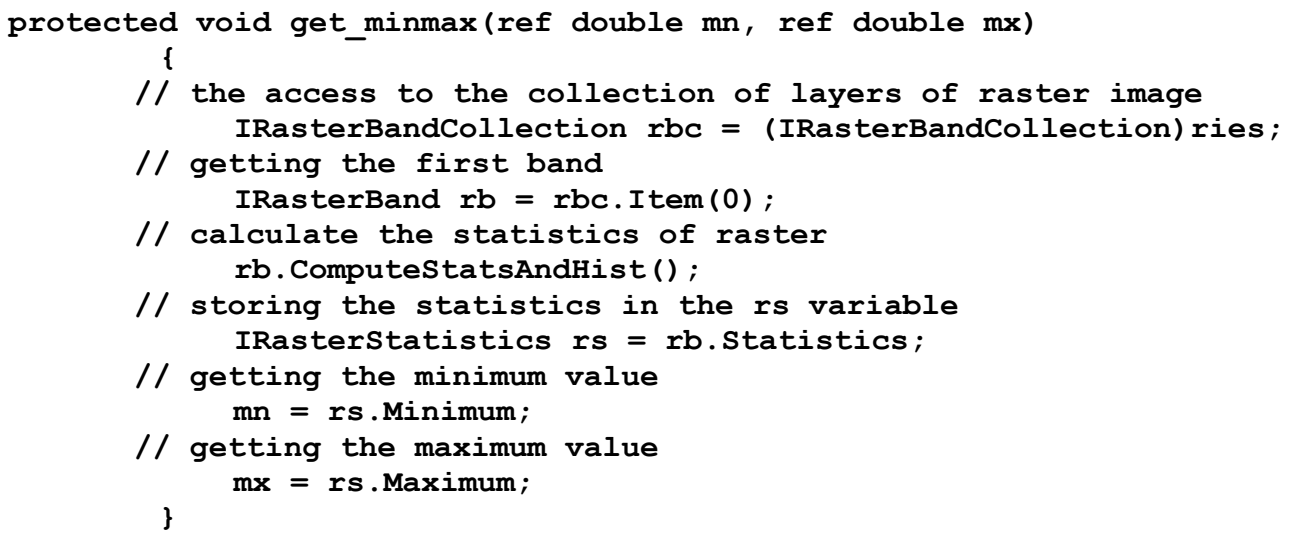

Simbologie_Raster_Interval method aims to symbolize the raster, so that the pixels to be colored according with the values membership to a range of values. In this way, the user can specify the number of groups, namely the number of intervals that have to be generated, and the pixels from each group will be separately colored. This view allows the user to mesh the raster, in terms of colors, so that the diversity of matrix of pixels values will be displayed, by using fewer colors. This method has to surprise important changes in density within the geographical area studied. The method gets the number of groups $(z)$, which is the number of intervals of values that has to be generated, and vsup parameter given by a symbolic constant which indicate the measure unit used in the labeling. Suprafata enumerative constant consists in 
the following constants: public enum Suprafata $\{$ M_patrati, KM_patrati $\}$;

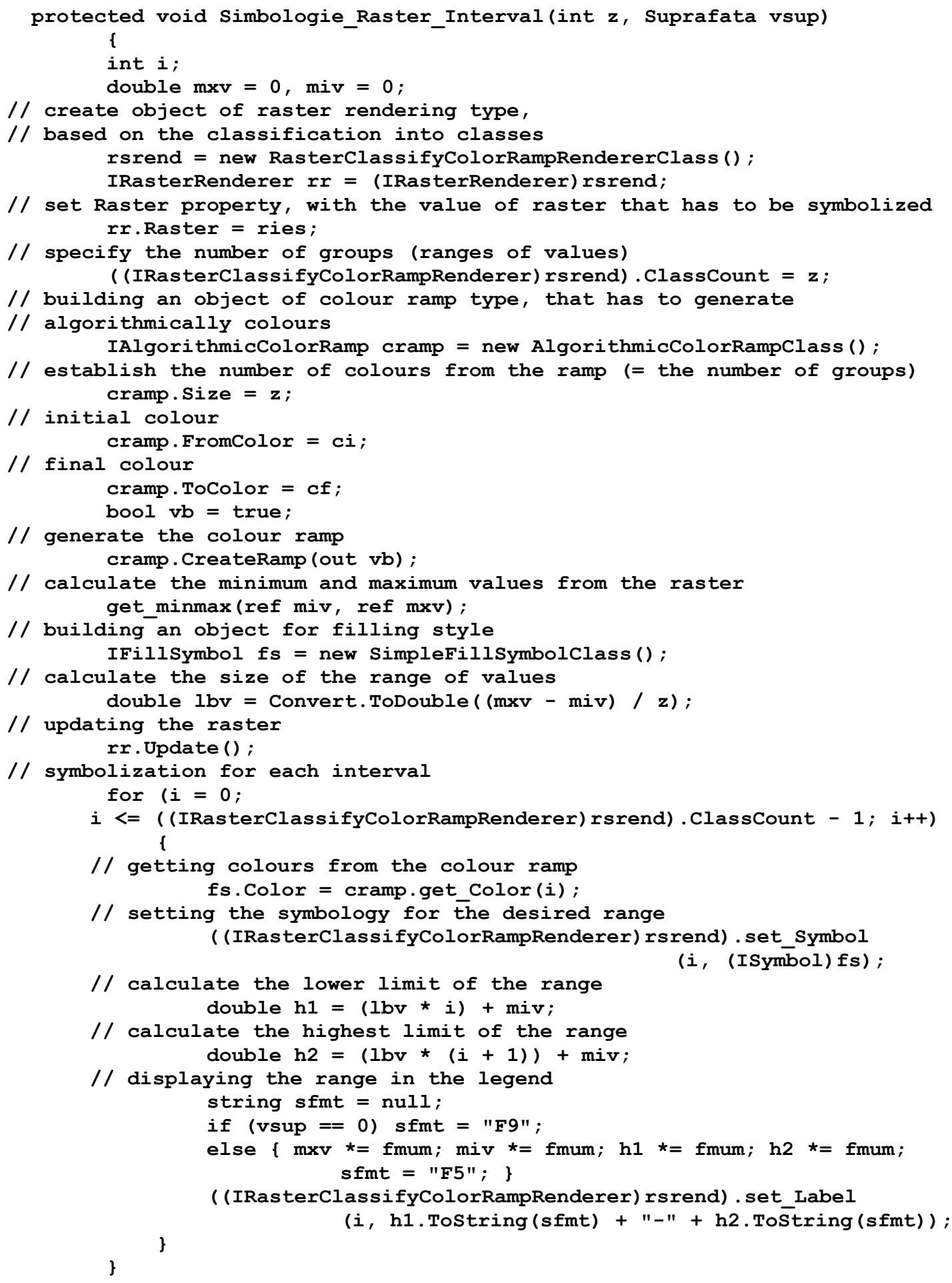

Afisare_Discreta method actually displays ues. It uses csup parameter, for the measure the symbolized raster by $n r g$ classes of val- unit used in labeling.

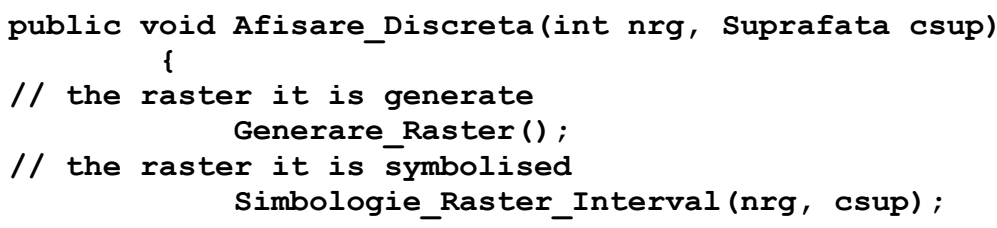




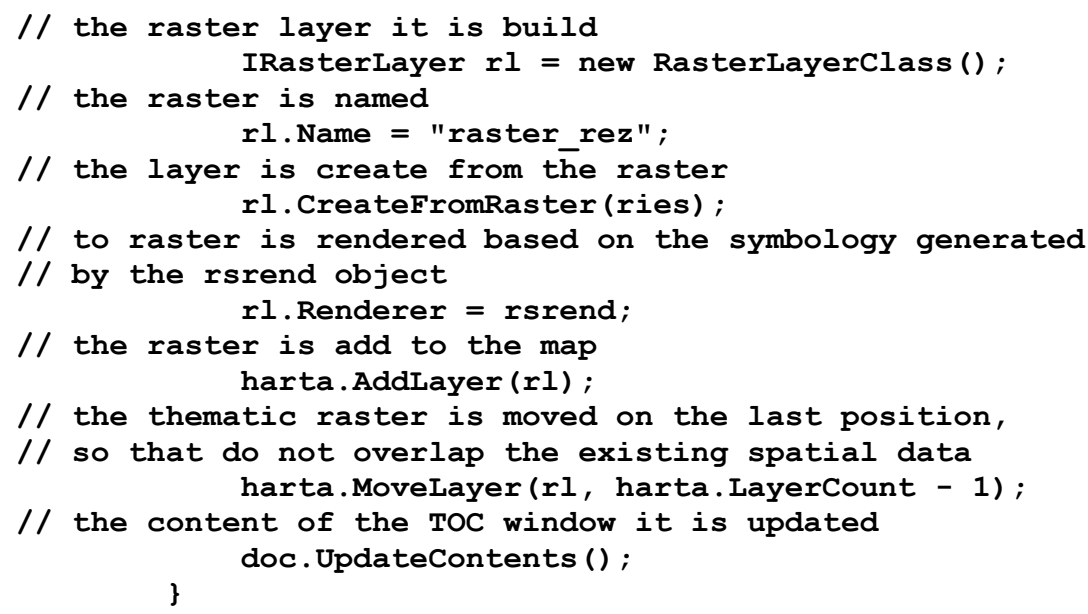

Another way to display the raster, defined inside the class, uses a continuous color pixel, and the matrix values have to be scaled on the color ramp used.

Simbologie_Raster_Rampa method achieves this by defining extreme colors of the color ramp, and by obtaining minimum and maximum values of the generated raster. The method receives as parameter the measure unit used to express the numerical values of the legend (vsup).

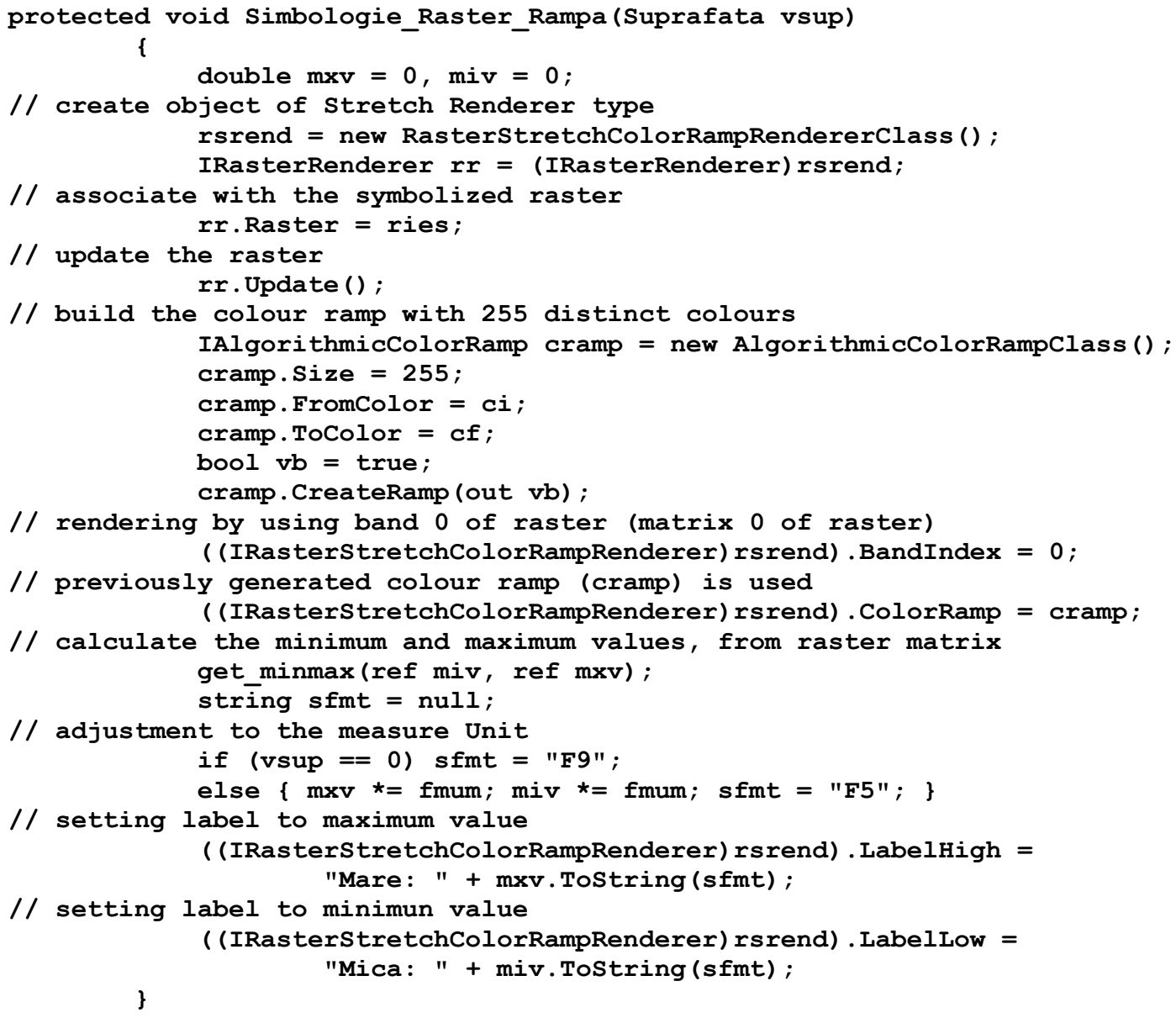

Afisare_Continua method generates a layer based on symbolized raster, and includes it to be viewed into the map. The parameter of this method specifies also the measure unit used to express numerical values of the legend. 


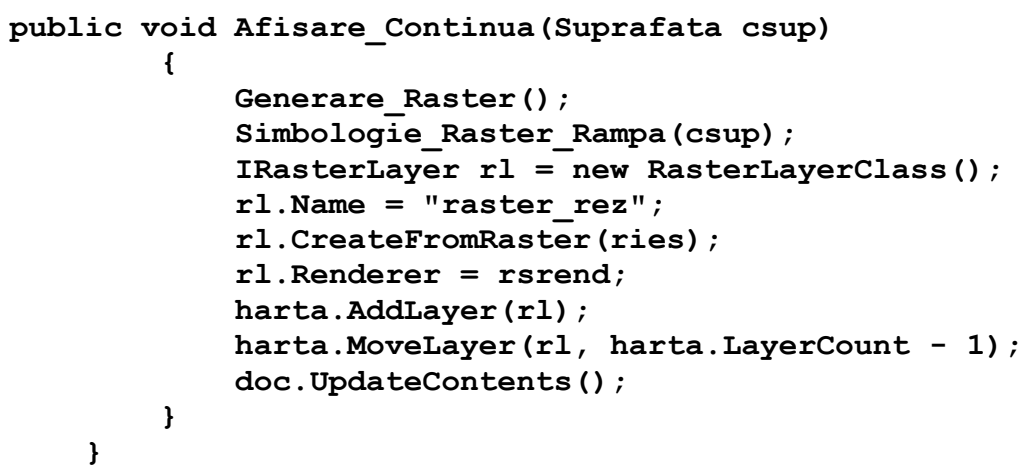

The derived classes implement specific elements to generate raster, and to highlight spatial data densities, function on their type. Thus, $R S \_$Densitate_Linii class is used to determine the density of spatial data of line type, while RS_Densitate_Puncte type is used for the point type data. In both classes the pure virtual method of abstract class (Generare_Raster) is defined to generate the appropriate raster, corresponding to the type of spatial data.

The $R S \_$Densitate_Linii class content is the following:

class RS_Densitate_Linii : RS_Densitate

\{

// the constructor that calls the base constructor and sets

// adjustment value according to the specified measurement unit public RS_Densitate_Linii (IMxDocument phdoc) : base (phdoc)

$\{$ fmum $=1000 ;\}$

// raster generation method overloading

protected override void Generare_Raster()

\{

// identify the layer for which the raster has to be generate

if (numel $!=$ null)

\{

int $i$;

for ( $i=0 ; i<$ harta. LayerCount; $i++$ )

if (harta.get Layer(i). Name $==$ numel)

\{

1_dr = harta.get_Layer(i)

break;

\}

if ( $i==$ harta. LayerCount)

throw new Dens_Ex("Nu exista layer-ul:" + numel);

else throw new Dens_Ex("Nu a fost precizat layer-ul!") ;

IFeatureLayer $\mathrm{fl}=$ (IFeatureLayer) 1 dr;

// testing the type of geometry for spatial data

if ( $f l$. FeatureClass. ShapeType!=esriGeometryType.esriGeometryPolyline) throw new Dens_Ex("Layerul nu e tip LINIE!!");

IGeoDataset gds $=$ (IGeoDataset) $\mathrm{fl}$. FeatureClass;

// building the object used to determine the density

IDensityOp rdc $=$ new RasterDensityOpClass ();

// building the object used to define the elements

// necessary to perform raster analysis IRasterAnalysisEnvironment renv = (IRasterAnalysisEnvironment) rdc;

// setting workspace renv. OutWorkspace $=$ ws

// setting raster cell size

object ocels = cels;

renv. SetCellsize

(esriRasterEnvSettingEnum.esriRasterEnvValue, ocels);

// define locations in which the raster has to be generate

IEnvelope env = new EnvelopeClass ();

env.XMin $=\mathrm{fl}$. AreaOfInterest.XMin

env.XMax $=\mathrm{fl}$.AreaOfInterest. XMax; 


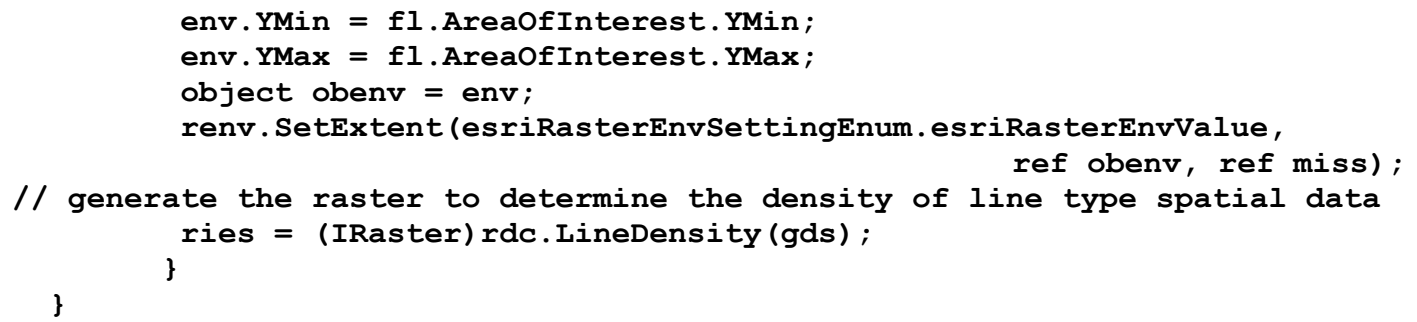

In the code sequence we can observe that certain exceptions are thrown through the Dens_Ex class. This class is derived from
Exception class and has the following content:

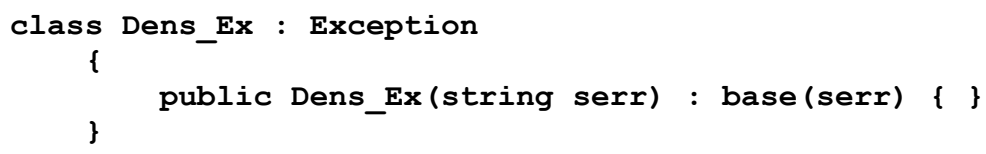

To test the functionality of generate colors in the following space: from $R S \_$Densitate_Linii class we used the themat- white - rgb $(255,255,255)$, which is associatic layer, with Romania's roads named ed with minimum value of the raster till red drumuri, the working space is given by $\operatorname{rgb}(255,0,0)$.

FileGeoDatabase which is stored in: The code sequence is: $d: \bigvee H \_d e n s i t a t e \bigvee b d s . g d b$. The color ramp used

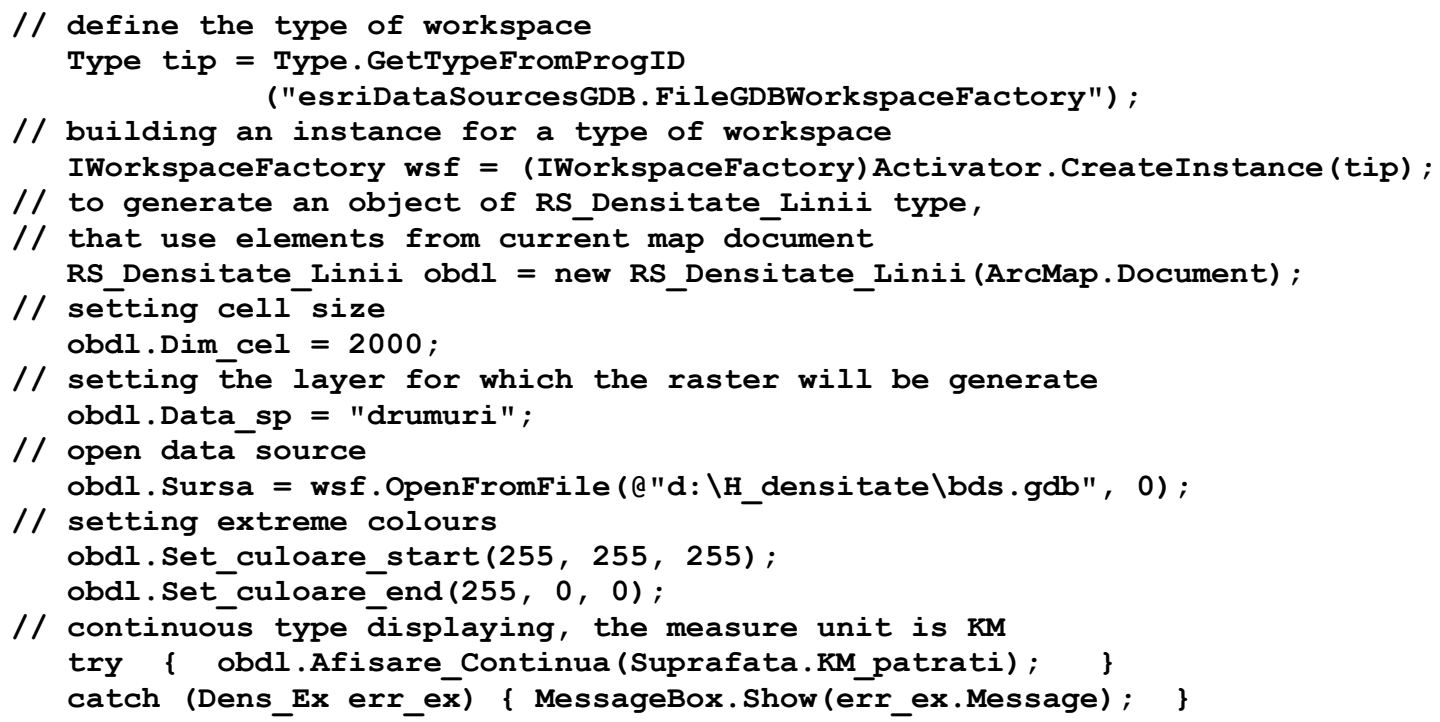

The code execution generates and allows displaying a raster, as presented in figure 3. The color space is scaled and it is associated with pixels having the values between the two extremes. In figure 3 we can observe that areas with a higher density of roads are intensely colored with red color, while other areas are colored with a more diluted red, so that no roads in an area, causes it to be colored in white. The density is calculated as the sum of the length of roads, per unit area. Being a map with geographical coordinates of projection type, the considered measure units could be meters (by default) or kilometers. 


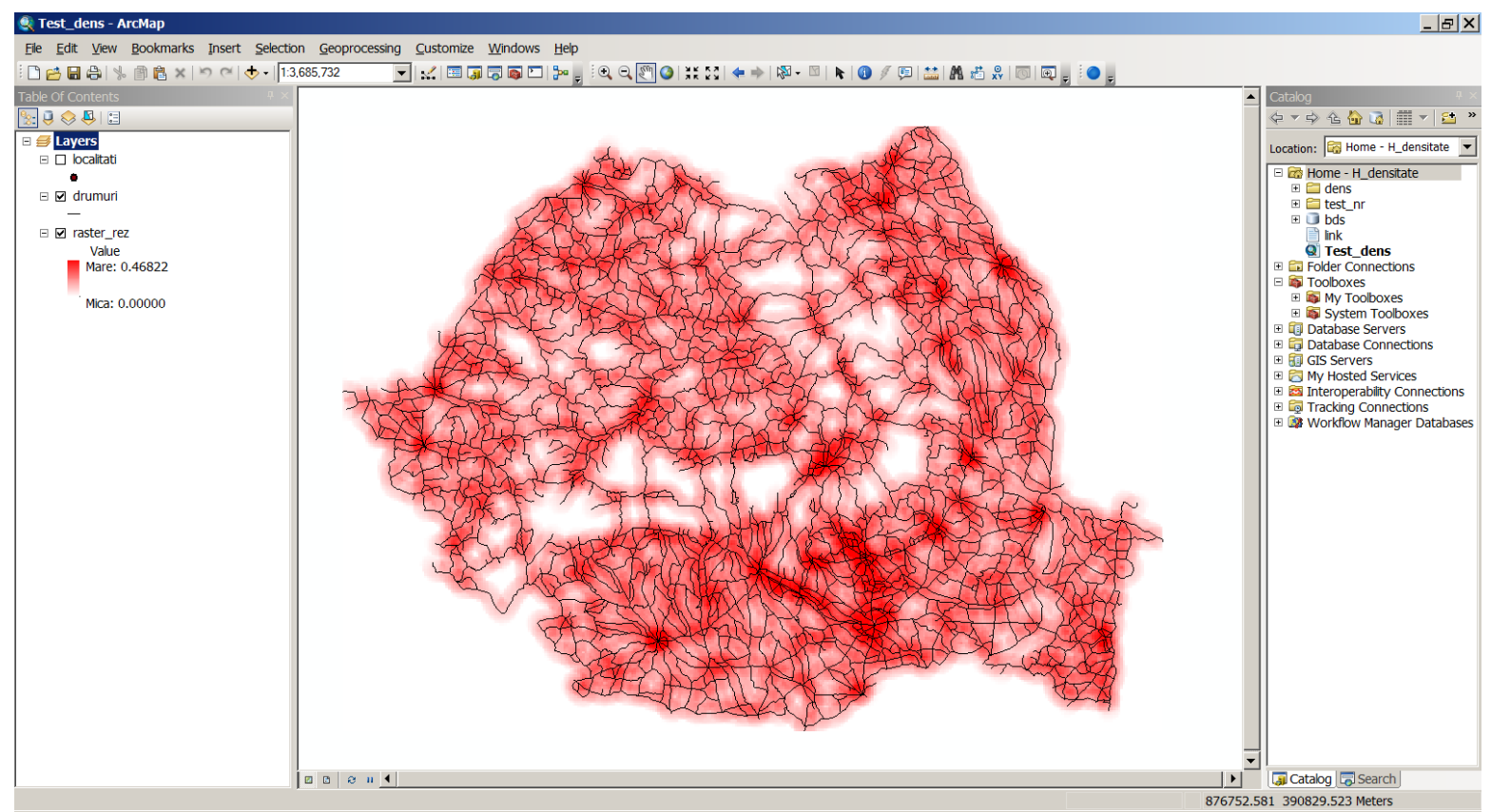

Fig. 3. Symbology used to display continuous density

RS_Densitate_Puncte class has similar content, but it has one additional parameter indicating the radius used to identify a circular area around a point, which is taken as a ref- erence area to calculate the density. A point is, in fact, a cell that has an associated dimension.

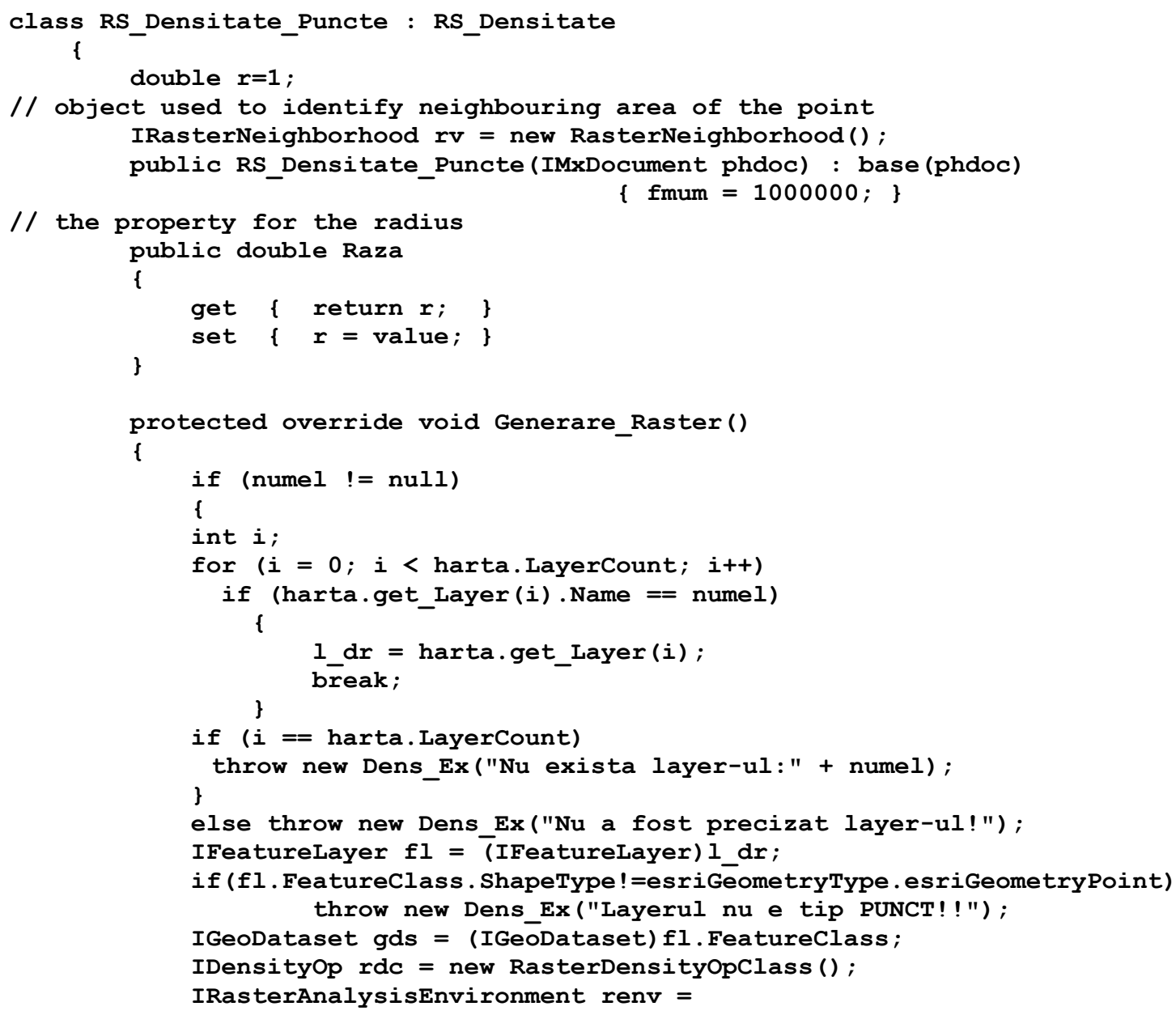


(IRasterAnalysisEnvironment) rdc;

renv. OutWorkspace $=$ ws;

object ocels = cels;

renv.SetCellsize (esriRasterEnvSettingEnum.esriRasterEnvValue,

IEnvelope env = new EnvelopeClass ();

ocels);

env.XMin $=\mathrm{fl}$.AreaOfInterest.XMin;

env.XMax $=\mathrm{fl}$. AreaOfInterest. XMax;

env.YMin $=\mathrm{fl}$.AreaOfInterest. YMin;

env. YMax $=\mathrm{fl}$.AreaOfInterest. YMax;

object obenv = env;

renv. SetExtent (esriRasterEnvSettingEnum.esriRasterEnvValue,

ref obenv, ref miss);

// area of circular neighbouring with the radius $r$, in map units

rv.SetCircle ( $r$, esriGeoAnalysisUnitsEnum.esriUnitsMap);

// generate the raster to determine the density of spatial data of point type ries $=$ (IRaster) rdc. PointDensity (gds, rv);

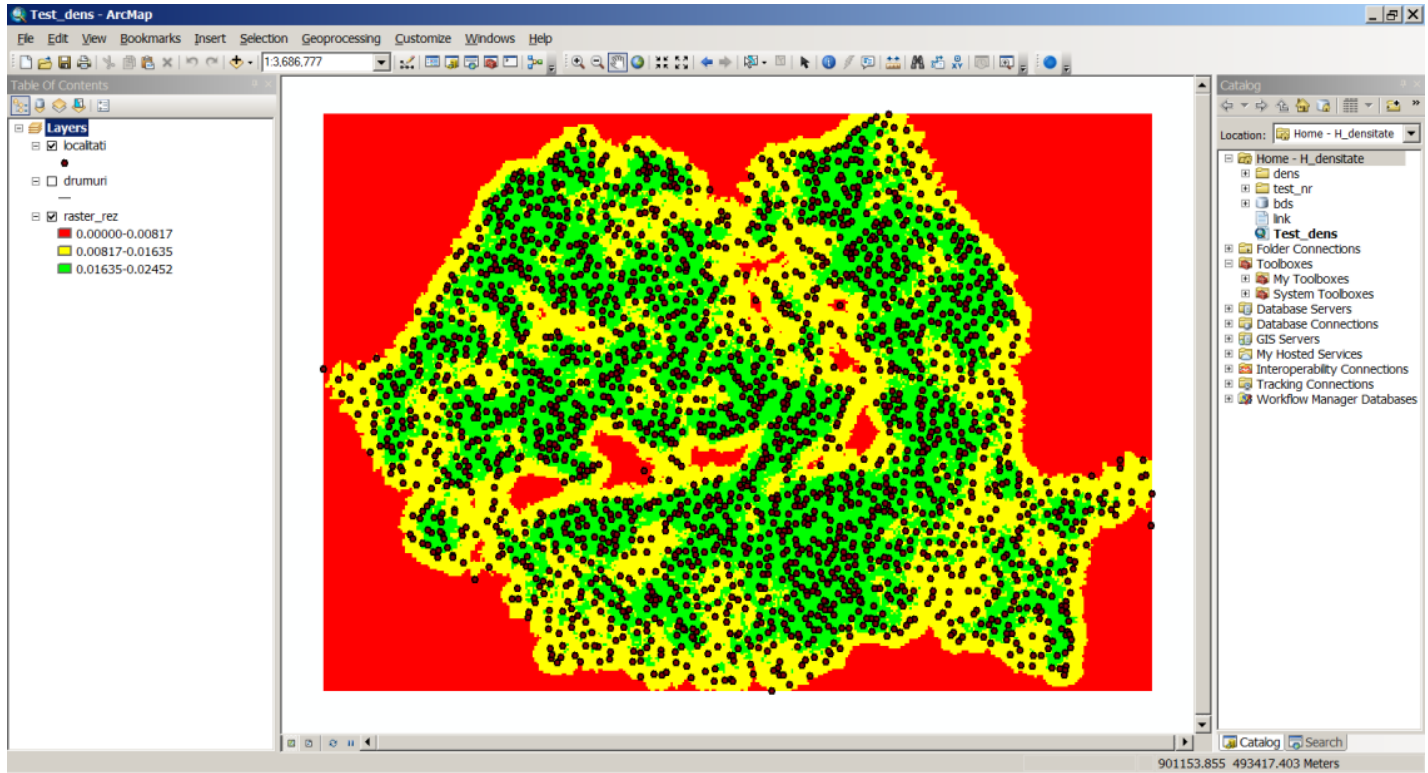

Fig. 4. Symbology used to display the density, by using three groups of values

To display the raster from the Figure 4, is used the method for discreet displaying obdp.Afisare_Discreta Suprafata.KM_patrati), which indicate three classes of values; $o b d p$ is the $R S \_D e n s i t a t e \_P u n c t e$ object.

\section{The Case Study}

The raster base solutions available to determine the density of points of interest could be used in various economic fields. These could be applied to identify the target study areas of interest, for in deep studies in various economic analyses, such as: to identify the best location for establishing a new business, function on the businesses developed in the proximity; to identify the location of one deposit-centre for a company with a lot of subsidiaries in one geographical area; to identify cluster based economic agglomerations function on the location of possible actors of the cluster [7], and on the transport infrastructure available to connect them.

Spatial data generated by GIS can be used in other various areas like in the hybrid support systems [8] in order to ensure effective support for smart business decision-making processes, in analysis the competition between various regions [9], and to classify the geographical areas function on population structure as in [10].

To demonstrate the utility and the functional- 
ity of proposed solutions, in the paper, we are trying to identify the cluster type economic agglomerations, in the pharmaceutical industry of Romania. To accomplish this task we have to identify the agglomerations of companies acting in pharmaceutical industry, located in a geographical proximity as the first step, and the second step supposes to identify the geographical areas with a good agglomeration of transport facilities (roads).

In the figure 5 , the geographical distribution of companies performing in pharmaceutical industry of Romania is figured, by using points. On the same map, the layer with the available roads has been represented.

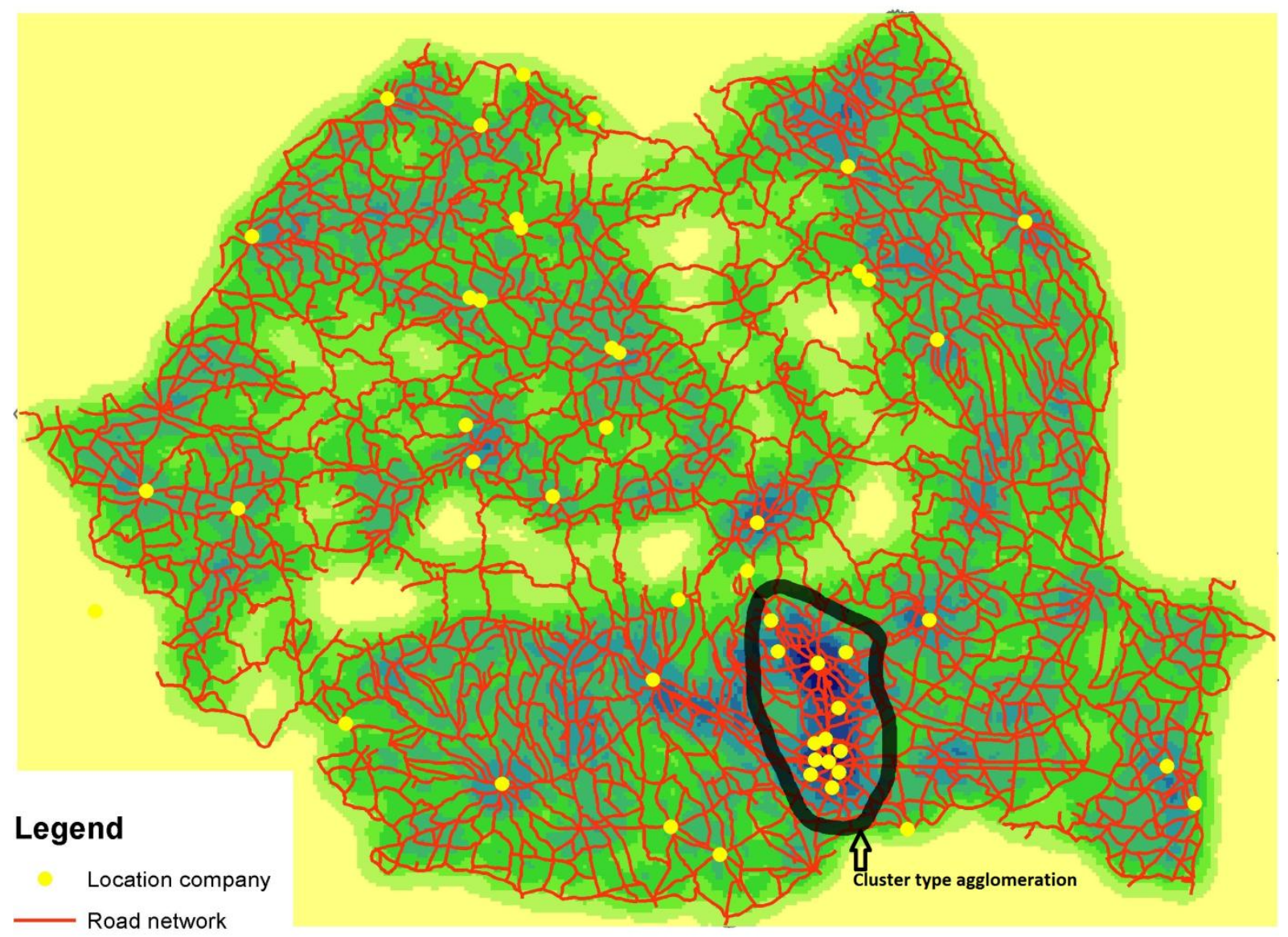

Fig. 5. Spatial agglomerations in pharmaceutical industry of Romania

By using the software components developed, as they are presented in this paper in the previous sections, the density of companies (points) and the density of roads available to link them (lines) have been identified, in each geographical area. The results are figured on the same map, and are presented in figure 5.

A strong economic agglomeration of companies acting in pharmaceutical industry could be identified in the South part of Romania, in Bucharest-Ilfov-Prahova area. Even there are a lot of agglomerations of companies performing in this field, in various geographical areas, because there is not good enough transport infrastructure available, to link them, we can conclude that there are not good conditions to identify a successful economic cluster in pharmaceutical field, in these regions.

\section{Conclusions}

To display in GIS the economic phenomena, namely the statistical data, both symbologies based on spatial data and raster symbology can be used. Symbologies associated with spatial data are limited to their location, while raster based symbologies facilitate the 
definition of areas that are strictly related to the phenomenon of spatiality.

The raster based solutions facilitate to integrate the spatial and economic data and to perform analysis like the spatial distribution of economic issues in space. The software components presented in the paper automate the processing of both economic and spatial data and generate the appropriate maps containing both types of data.

\section{Acknowledgement}

This work was supported by the project "Performance and excellence in postdoctoral research in Romanian economics science domain", POSDRU contract No. 89/1.5/S/59184.

\section{References}

[1] E.-L. Glaeser, H.-D. Kallal, J.-A. Sheinkman and A. Sheifler, "Growth in Cities", Journal of Political Economics, no. 100, 1992, pp. 1126-1152.

[2] K.-T., Chang, "Programming ArcObjects with VBA", CRC Press, 2008.

[3] M. Dardala, A. Reveiu, "Techniques for Statistical Data Visualization in GIS", Informatica Economică Journal, vol 15, no. 3, 2011, pp. 72-79.
[4] R., Burke, "Getting to Know ArcObjects: Programming ArcGIS with VBA", ESRI Press, 2003.

[5] V. D. Mihon, A. Minculescu, V. Colceriu and D. Gorgan, "Diagramatic description of distributed spatial data processing", Revista Romana de Interactiune OmCalculator, vol. 5(3), 2012, pp. 59-80.

[6] ***, Exploring ArcObjects, ESRI Press, 2002.

[7] Boja, C., "Clusters Models, Factors and Characteristics", International Journal of Economic Practices and Theories, no. 1 (2011), pp. $34-43$.

[8] C. Brandas, "Hybrid Support Systems: a Business Intelligence Approach", Journal of Applied Business Information Systems, 2(2), pp: 57 - 62, 2011.

[9] R. C. Mușetescu, "The Economics of State Aid Control in the European Union during the Financial Crisis: The Challenge for a Post-Crisis Rhetoric." Theoretical and Applied Economics 6 (XIX), 2012: 175-184.

[10] C. Herţeliu, M.A. Farcasanu, "The Religious Affiliation Structure of the Villages from Romania", Journal of Applied Quantitative Methods, no. 1, 2011, pp. 121-128.

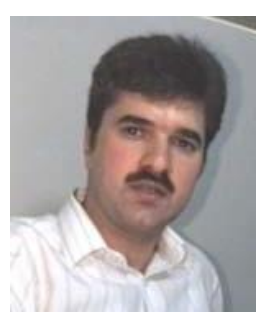

Marian DÂRDALĂ has graduated the Faculty of Cybernetics, Statistics and Economic Informatics. He holds a PhD diploma in Economic Cybernetics and Statistics. He is professor in Economic Informatics field and branches within Department of Economic Informatics at faculty of Cybernetics, Statistics and Economic Informatics from Academy of Economic Studies. He is the author and co-author of 11 books and over 50 articles in journal and proceedings of national and international conferences, symposiums, workshops in the fields of multimedia, human computer interaction - HCI, GIS, data bases and object oriented programming.

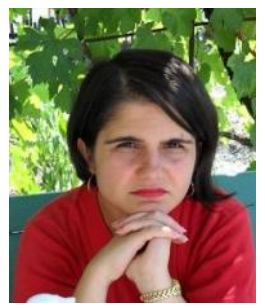

Adriana REVEIU has graduated the Faculty of Cybernetics, Statistics and Economic Informatics. She holds a PhD diploma in Economic Cybernetics and Statistics. She is associate professor in Economic Informatics field and branches within Department of Economic Informatics at faculty of Cybernetics, Statistics and Economic Informatics from Bucharest Academy of Economic Studies. She is the author and co-author of 10 books and over 50 articles in journal and proceedings of national and international conferences, symposiums, workshops in the fields of regional development, data management, multimedia and learning systems. 